\title{
Das allgemeine Exponential-Verbundgraphentheorem
}

\author{
Paul Ziesche \\ Abteilung für Theoretische Physik \\ Pädagogisches Institut Dresden \\ Eingegangen am 1. Februar 1967
}

\begin{abstract}
General theorems concerning reordering of products of exponential functionals are formulated and proved. Their applicability in different fields of many particle theory is mentioned.
\end{abstract}

\section{Einleitung}

In der Vielteilchentheorie kommt bei der (störungstheoretischen) Behandlung der Teilchenwechselwirkung wiederholt die rein mathematische Aufgabe vor, in Ausdrücken vom Typ

$$
e^{g\left(\frac{\delta}{\delta \varphi}\right)} e^{f(\varphi)}, \quad e^{g\left(\frac{\delta}{\delta \psi}, \frac{\delta}{\delta \bar{\psi}}\right)} e^{f(\bar{\psi}, \psi)}, e^{g\left(\frac{\delta}{\delta \psi}, \frac{\delta}{\delta \varphi}, \frac{\delta}{\delta \bar{\psi}}\right)} e^{f(\bar{\psi}, \varphi, \psi)}
$$

die Differentionen auszuführen, d. h. die Differentialoperatoren nach rechts zu tauschen. Erstmalig traten solche Aufgaben bei der Entwicklung relativistischer Quantenfeldtheorien im Zusammenhang mit dem von Hori, Anderson und Matsubara kompakt formulierten Wickschen Theorem [1-5] auf, später auch bei der formalen Lösung von Funktionaldifferentialgleichungen für die zuerst von BogoLJuBow und SchwINGER [6-8] eingeführten erzeugenden Funktionale [9-14]. Mit der Übertragung quantenfeldtheoretischer Methoden auf nichtrelativistische Vielteilchensysteme sowie auf Probleme der quantenmechanischen und klassischen ${ }^{1}$ Gleichgewichts- und Nichtgleichgewichts ${ }^{2}$ Statistik wurden auch in diesen Gebieten Spezialfälle der genannten Aufgaben immer wieder behandelt. Jedesmal traten als Ergebnis sog. Verbundgraphentheoreme auf, nach denen sich die aus den Bauelementen von $g(.$.$) bzw. f(\ldots)$ aufgebauten, aber nicht faktorisierten Terme

${ }^{1}$ In der klassischen Gleichgewichts-Statistik wird dies durch Anwendung und Ausbau des zuerst von ScHÖNBERG entwickelten klassischen BesetzungszahlFormalismus ermöglicht $[15,16]$.

2 In der quantenmechanischen und klassischen Nichtgleichgewichts-Statistik wird dies durch Überführung der von-Neumann- bzw. Liouville-Gleichung in die Bewegungsgleichungen für die erzeugenden Funktionale der reduzierten Dichtematrizen bzw. Verteilungsfunktionen erreicht [17-19].

21 Commun. math. Phys., Vol. 5 
wieder in Exponentialform ansammeln. Im folgenden wird ein alle diese Spezialfälle enthaltendes allgemeines Exponential-Verbundgraphentheorem bewiesen, wobei der Beweis überraschenderweise formal auf entsprechende Aufgaben der Mayerschen Clustertheorie [20, 21] zurückgeführt werden kann, die in der klassischen Gleichgewichts-Statistik entwickelt wurde, lange bevor die quantenfeldtheoretischen Methoden zur Verfügung standen und ihre Anwendbarkeit auch auf die klassische Gleichgewichts- und Nichtgleichgewichts-Statistik bekannt war [15-19]. Ubrigens sind auch die Operatorenumordnungen vom obengenannten Typ, die beim klassischen Grenzübergang entweder mit den (in der Kohärenztheorie der Laser auftretenden) Zuständen maximaler Bestimmtheit oder mit der Weyl-Korrespondenz und der Wigner-Transformation zusammenhängen.

Die Formeln sind abschnittsweise durchnumeriert und werden zusammen mit den Abschnittsnummern zitiert. Zur formalen Vereinfachung wird in Anlehnung an Symanzik [10] für Mehrfachintegrale eine abkürzende Schreibweise benutzt.

\section{Allgemeine Kontraktionsaufgabe mit einem Operator}

\subsection{Formulierung der Aufgabe}

Hier wird die Aufgabe

$$
e^{g\left(\frac{\delta}{\delta \varphi}\right)} e^{f(\varphi)}=e^{\sum \frac{1}{s !} \theta_{s} \frac{\delta^{s}}{\delta \varphi^{s}}} e^{\sum \frac{1}{r !} \varphi^{r} f_{r}}
$$

behandelt. In den Taylor/Volterra-Reihen der Funktionale $g(. .$.$) und$ $f(. .$.$) wird in Anlehnung an die Einsteinsche Summenregel konsequent$ die Abkürzung

$$
\begin{aligned}
\varphi^{r} f_{r} & \equiv \int d x_{1} \varphi\left(x_{1}\right) \ldots \int d x_{r} \varphi\left(x_{r}\right) f_{r}\left(x_{1}, \ldots, x_{r}\right) \\
& =\int d x_{1} \ldots \int d x_{r} f_{r}\left(x_{1}, \ldots, x_{r}\right) \varphi\left(x_{1}\right) \ldots \varphi\left(x_{r}\right) \equiv f_{r} \varphi^{r}
\end{aligned}
$$

benutzt, wobei $x$ gegebenenfalls einen ganzen Satz von Variablen z. B. $(\mathfrak{r}, t)$ oder $(\mathfrak{r}, \mathfrak{p})$ usw. bedeutet. Die Koeffizientenfunktionen $f_{r}(\ldots)$ und $g_{s}(\ldots)$ mögen entsprechend den physikalischen Aufgabenstellungen so beschaffen sein, daß sie nicht in Faktoren mit voneinander unabhängigen Variablengruppen zerfallen (Verbundeigenschaft). Die Entwicklung von $g(.$.$) legt ein schrittweises Vorgehen nahe:$

$$
e^{g\left(\frac{\delta}{\delta \varphi}\right)} e^{f(\varphi)}=\cdots e^{\frac{1}{3 !} g_{\mathbf{z}} \frac{\delta^{3}}{\delta \varphi^{3}}} e^{\frac{1}{2 !} g_{2} \frac{\delta^{2}}{\delta \varphi^{2}}} e^{g_{1} \frac{\delta}{\delta \varphi}} e^{f(\varphi)} \equiv \cdots e^{L} e^{K} e^{J} e^{f(\varphi)} .
$$

Auf diese Weise werden nacheinander die „Einer“-, ,Zweier"-, „Dreier“Kontraktionen usw. hergestellt. 


\subsection{Herstellung der Einer- und Zweier-Kontraktionen}

Der erste Schritt ist mit der Taylor/Volterra-Entwicklung trivial:

$$
e^{J} e^{f(\varphi)}=e^{g_{1} \frac{\delta}{\delta \varphi}} e^{f(\varphi)}=e^{f\left(\varphi+g_{1}\right)} .
$$

In diesem so entstandenen Ausdruck verknüpft der Operator der ZweierKontraktionen $K \equiv(1 / 2 !) g_{2} \delta^{2} / \delta \varphi^{2}$ beim nächsten Schritt in (11.3) alle möglichen Paare $\varphi_{1} \equiv \varphi\left(x_{1}\right), \varphi_{2} \equiv \varphi\left(x_{2}\right)$ :

$$
\begin{aligned}
\widehat{\varphi}_{1} \varphi_{2} \equiv K \varphi_{1} \varphi_{2}=\frac{1}{2}\left\lceil g_{2} \frac{\delta^{2}}{\delta \varphi^{2}} \varphi_{1} \varphi_{2}\right. & =g_{2}(1,2), \\
\operatorname{da}\left[\frac{\delta}{\delta \varphi\left(x_{1}\right)}, \varphi\left(x_{2}\right)\right] & =\delta\left(x_{1}-x_{2}\right) .
\end{aligned}
$$

Daß in (11.3) jede Potenz $K^{n}$ entsprechend der Exponentialform exp $K$ mit einem Faktor $1 / n$ ! versehen ist, bedeutet, daß jede Kontraktion eines Operatorenprodukts, d. h. jede Verteilung von Haken genau einmal vorkommt.

Zweckmäßig stellt man nun zunächst nur innerhalb von $f\left(\varphi+g_{1}\right)$ alle Zweierkontraktionen her

$$
e^{K} f\left(\varphi+g_{1}\right) \equiv \bar{f}(\varphi)
$$

und benutzt das dabei entstehende, von $f\left(\varphi+g_{1}\right)$ natürlich verschiedene und hier mit $\vec{f}(\varphi)$ bezeichnete Funktional von $\varphi$ für die weiteren Kontraktionen als Bauelement. Beim vollständigen Kontrahieren eines Produkts von zwei verschiedenen Funktionalen $f_{1}(\varphi)$ und $f_{2}(\varphi)$

$$
e^{K} f_{1} f_{2}=e^{i, j=1} \sum_{i j}^{2} K_{1} f_{2}=e^{K_{12}+K_{21}} \bar{f}_{1} \overline{f_{2}}
$$

wird der Kontraktionsoperator durch die Produktregel der Differentialrechnung automatisch in eine Summe zerlegt

$$
\begin{aligned}
K & =\frac{1}{2} \overrightarrow{\varphi \varphi} \frac{\delta^{2}}{\delta \varphi \delta \varphi}=\sum_{i, j} \frac{1}{2} \overrightarrow{\varphi \varphi} \frac{\delta}{\delta \varphi_{i}} \frac{\delta}{\delta \varphi_{j}} \\
& =\sum_{i} \frac{1}{2} \hat{\varphi \varphi} \frac{\delta}{\delta \varphi_{i}} \frac{\delta}{\delta \varphi_{i}}+\sum_{i<j} \mathscr{\varphi \varphi} \frac{\delta}{\delta \varphi_{i}} \frac{\delta}{\delta \varphi_{j}} \equiv \sum_{i} K_{i i}+\sum_{i<j} K_{i j},
\end{aligned}
$$

wobei die mit einem Index $i$ bzw. $j$ versehenen Differentialoperatoren nur auf den zugehörigen Faktor $f_{i}$ bzw. $f_{j}$ wirken. Daher stellt $K_{11}$ nur die Kontraktionen innerhalb $f_{1}$ her, $K_{22}$ nur innerhalb $f_{2}$. Zwischen den so entstehenden Termen $\bar{f}_{1}, \bar{f}_{2}$ stellt dann weiter $K_{12}$ alle möglichen Verknüpfungen von Operatorenpaaren her, von denen ein Operator zu $\bar{f}_{1}$ und der andere zu $\bar{f}_{2}$ gehört. Je nachdem wieviele Kontraktionspartner in $\bar{f}_{1}$ und $\bar{f}_{2}$ noch zur Verfügung stehen, können zwischen $\bar{f}_{1}$ und $\bar{f}_{2}$ eine Kontraktion, zwei Kontraktionen usw. hergestellt werden, für die zusammenfassend die folgende Kennzeichnung durch eine dick gezeichnete $21^{*}$ 
Verknüpfung eingeführt wird:

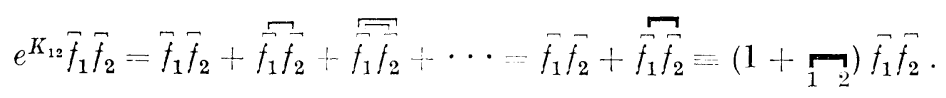

Damit ergibt sich für die Kontraktion mehrerer Faktoren

$$
e^{K} f_{1} \ldots f_{n}=e^{i<j} K_{i j} \vec{f}_{1} \ldots \bar{f}_{n}=\prod_{i<j}\left(1+\eta_{j}\right) \vec{f}_{1} \ldots \vec{f}_{n}=\sum_{n} \vec{f}_{1} \sqcap \ldots \overrightarrow{f_{n}} .
$$

In diesem verallgemeinerten Wickschen Theorem wird durch $\sum_{\sim}$ über alle möglichen (dicken) Kontraktionen zwischen den $\bar{f}_{i}$ summiert, wobei jedes $\bar{f}_{i}$ an beliebig vielen (dicken) Kontraktionen beteiligt sein kann.

Wie üblich gewinnt man auch hier einen besseren Überblick über die entstehenden Terme durch Einführung von Clustern

$$
\vec{f}_{i} \equiv \rho_{i} \quad \vec{f}_{i} \vec{f}_{j} \equiv i_{i} \text {. }
$$

Für die Kontraktion von drei Faktoren ergibt sich z. B.

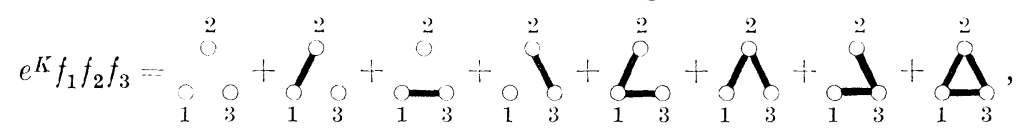

woraus für identische Funktionale $f_{1}=f_{2}=f_{3}$ die einfachere Beziehung

$$
e^{K} \frac{f^{3}}{3 !}=\frac{1}{3 !}(0 \circ 0)+\frac{1}{2 !}(0 \bigcirc)+\frac{1}{2 !}-\infty+\frac{1}{3 !} \boldsymbol{\Delta}
$$

entsteht. Allgemein entsteht bei Anwendung dieser Regeln auf $f_{1} \ldots f_{n}$ bzw. $f^{n}$ eine Summe von Produktclustern, die jeweils aus einfacheren Clustern, den sog. Verbundclustern (linked cluster) aufgebaut sind.

Man bekommt alle zu $f_{1} \ldots f_{n}$ gehörigen Cluster, indem man wiederholt jeweils $n$,Punkte“ $\circ$ zeichnet, diese durchnumeriert und nun nach dem verallgemeinerten Wickschen Theorem (7) in jedes dieser $n$-tupel von Punkten auf alle möglichen verschiedenen Weisen Verknüpfungen einzeichnet. Damit entsteht die Summe aller voneinander verschiedenen numerierten Cluster (jeder genau einmal). Diese Summe der numerierten Cluster erhält man daher auch, indem man alle voneinander verschiedenen, aber unnumerierten Cluster zeichnet, anschließend irgendeine Numerierung der Punkte vornimmt und nun in jedem dieser numerierten Cluster von den insgesamt $n !$ möglichen Permutationen der PunktNummern alle diejenigen einträgt, die verschiedene numerierte Cluster liefern (siehe z. B. (9)). Nur bei einer gewissen Zahl $z$ der $n$ ! Permutationen entstehen aus einem numerierten Cluster wirklich voneinander verschiedene numerierte Cluster, die natürlich auf Grund der Konstruktion alle gleiche Struktur aufweisen, während bei den restlichen der $n$ ! Permutationen aus jedem der $z$ so gewonnenen verschiedenen numerierten Cluster gleicher Struktur auf Grund ihrer topologischen Symme- 
trien jeweils $g$ gleiche numerierte Cluster entstehen, z. B.

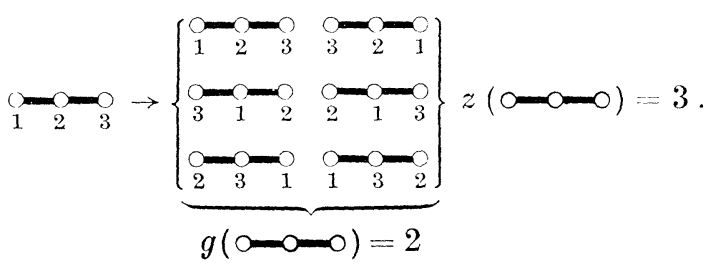

Daher gilt ganz allgemein $z g=n$ ! für jede Cluster-Struktur mit $n$ Punkten. In der Tabelle sind weitere Beispiele für die SymmetrieFaktoren von Clustern angegeben. Übrigens stellt $z$ für jeden Cluster zugleich die Zahl derjenigen Zerschneidemöglichkeiten des zugehörigen symmetriereichsten Cluster gleicher ,Ordnung“ $n$ (in dem jeder mit jedem Punkt verbunden ist und der daher den Symmetriefaktor $g=n$ ! besitzt) dar, die zu dem betreffenden Cluster führen. Überhaupt entstehen wie bei allen Cluster- und Graphen-Entwicklungen aus den

Tabelle 1. Clusterstrukturen und itre Symmetriefaktoren

\begin{tabular}{c|c|c}
\hline Cluster-Struktur & $g$ & $z$ \\
\hline 0 & 1 & 1 \\
\hline & 2 & 1 \\
\hline \\
\hline
\end{tabular}

symmetriereichsten Verbundclustern bzw. -graphen alle übrigen Cluster bzw. Graphen durch sukzessives Zerschneiden. Wie die in der Tabelle 
angegebenen Beispiele zeigen, entstehen offenbar genau die von der klassischen Gleichgewichtsstatistik her bekannten Mayerschen Cluster $[20,21]$. Das ist auch nicht weiter verwunderlich, da die Konstruktion der Cluster gemäß (7) in beiden Fällen genau die gleiche ist.

Bei dem in $\exp K \exp f$ benötigten Übergang zu identischen (sozusagen ununterscheidbaren) Funktionalen $f_{1}=f_{2}=\cdots$ werden die jeweils $z$ verschiedenen numerierten Cluster gleicher Struktur identisch, d. h. erhalten gleiche mathematische Bedeutung. Um den Beitrag der $z_{\gamma}$ numerierten Cluster gleicher Struktur $\gamma$ wiederzugeben, genügt es daher, den zugehörigen unnumerierten Cluster mit $z_{\gamma}$ zu multiplizieren:

$z_{\gamma} \mathbb{Z}_{\gamma}=z_{\gamma}(\tilde{f} \ldots \sqcap \bar{f})_{\gamma} \equiv n_{\gamma} ! \gamma \curvearrowright \gamma=\frac{1}{g_{\gamma}} \mathbb{Z}_{\gamma}=\frac{1}{g_{\gamma}}(\tilde{f} \ldots \sqcap \bar{f})_{\gamma}, z_{\gamma} g_{\gamma}=n_{\gamma} !$.

Das schraffierte Viereck steht symbolisch für irgendeine Clusterstruktur $\gamma$ der $\tilde{f}$. Im allgemeinen besteht nun ein beliebiger $\operatorname{zu}(\exp K) f^{n}$ beitragender Cluster aus einem Produkt der Verbundcluster $\gamma_{i}$, nämlich aus $n_{1}$ Verbundclustern $\gamma_{1}$, aus $n_{2}$ Verbundclustern $\gamma_{2}$ usw., so daß sich der Symmetriefaktor $g_{n_{1} n_{2} n_{3}} \ldots$ dieses Produktclusters $\gamma_{n_{1} n_{2}} \ldots$ in einfacher Weise auf die Symmetriefaktoren $g_{i}$ der konstituierenden Verbundcluster $\gamma_{i}$ zurückführen läßt:

$$
g_{n_{1} n_{2} \ldots}=n_{1} ! n_{2} ! \ldots g_{1}^{n_{1}} g_{2}^{n_{2}} \ldots \curvearrowright \gamma_{n_{1} n_{2} \ldots}=\frac{\gamma_{1}^{n_{1}}}{n_{1} !} \frac{\gamma_{2}^{n_{2}}}{n_{2} !} \cdots
$$

Damit liefern nun die Zweier-Kontraktionen insgesamt

$$
\begin{gathered}
e^{K} e^{f}=e^{K} \sum_{n} \frac{f^{n}}{n !}=\sum_{n_{1}, n_{2}, \ldots} \frac{\gamma_{1}^{n_{1}}}{n_{1} !} \frac{\gamma_{2}^{n_{2}}}{n_{2} !} \cdots=e^{\gamma_{1}+\gamma_{2}+\cdots}=e^{\gamma} \\
K=\frac{1}{2 !} g_{2} \frac{\delta^{2}}{\delta \varphi^{2}}
\end{gathered}
$$

mit

$$
\begin{aligned}
& \gamma(\varphi)=\sum_{i} \cdot \gamma_{i}(\varphi)=\sum_{i} \frac{1}{g_{i}}(\bar{f} \ldots \sqcap \bar{f})_{i} \\
& =\sum_{i} \frac{1}{g_{i}}{Z Z_{i}}=0+\frac{1}{2} \mathbf{g}+\frac{1}{3 !} \mathbf{d}+\frac{1}{2} \boldsymbol{g}+\frac{1}{4 !} \mathbf{X} \mathbf{8}+ \\
& \left.+\frac{1}{4} \square+\frac{1}{8} \mathbb{Z}+\frac{1}{2} \Omega+\frac{1}{3 !} \mathcal{g}+\frac{1}{2}\right\}+\cdots
\end{aligned}
$$

als Summe aller durch Zweier-Kontraktionen entstehenden Verbundcluster. 


\subsection{Herstellung der höheren Kontraktionen}

Beim nächsten Schritt in (11.3) verknüpft der Operator der DreierKontraktionen $L \equiv\left(1 / 3\right.$ !) $g_{3} \delta^{3} / \delta \varphi^{3}$ in dem entstandenen Ausdruck $\exp \gamma(\varphi)$ alle möglichen Tripel $\varphi_{1} \equiv \varphi\left(x_{1}\right), \varphi_{2} \equiv \varphi\left(x_{2}\right), \varphi_{3} \equiv \varphi\left(x_{3}\right)$ :

$$
\stackrel{\varphi_{1} \varphi_{2} \varphi_{3}}{=}=L \varphi_{1} \varphi_{2} \varphi_{3}=\frac{1}{3 !} g_{3} \frac{\delta^{3}}{\delta \varphi^{3}} \varphi_{1} \varphi_{2} \varphi_{3}=g_{3}(1,2,3) \text {. }
$$

Wieder werden zweckmäßig zunächst nur die Dreier-Kontraktionen innerhalb eines Funktionals $\gamma(\varphi)$ hergestellt

$$
e^{L_{1}} \gamma_{1}=e^{\frac{1}{3 !} \stackrel{\varphi}{\varphi} \varphi} \frac{\delta^{3}}{\delta \varphi \delta \varphi \delta \varphi} \gamma_{1}(\varphi) \equiv \boldsymbol{\gamma}_{1}(\varphi) \equiv \underset{1}{\otimes},
$$

dann in einem Produkt von zwei Funktionalen $\boldsymbol{\gamma}_{1}(\varphi)$ und $\boldsymbol{\gamma}_{2}(\varphi)$

$$
\begin{aligned}
& e^{L_{12}} \ddot{\gamma}_{1} \widetilde{\gamma}_{2}=e^{\frac{1}{2 !} \widetilde{\varphi \varphi \varphi}}\left(\frac{\delta^{2}}{\delta \varphi_{1}^{2}} \frac{\delta}{\delta \varphi_{2}}+\frac{\delta}{\delta \varphi_{1}} \frac{\delta^{2}}{\delta \varphi_{2}^{2}}\right) \widetilde{\gamma}_{1} \widetilde{\gamma}_{2}
\end{aligned}
$$

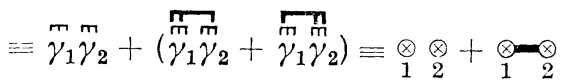

und schließlich in einem Produkt von drei Funktionalen $\mathbb{\gamma}_{1}(\varphi), \mathbb{\gamma}_{2}(\varphi)$ und $\dddot{\gamma}_{3}(\varphi)$

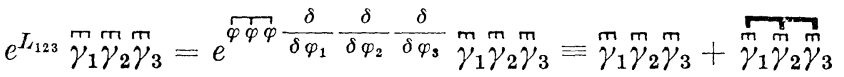

$$
\begin{aligned}
& \equiv \underset{1}{\otimes} \underset{2}{\otimes} \underset{3}{\otimes}+\overbrace{1}^{\otimes}
\end{aligned}
$$

Mit diesen Bauelementen (2), (3), (4) liefert die Produktregel, die auch hier $\delta / \delta \varphi$ in eine entsprechende Summe zerlegt, bei der Herstellung aller Dreier-Kontraktionen in $\gamma_{1} \gamma_{2} \gamma_{3}$ folgende Clustersumme:

$$
\begin{aligned}
& e^{L} \gamma_{1} \gamma_{2} \gamma_{3}=e^{\frac{1}{3 !} \Phi \varphi \gamma}\left(-\frac{\delta}{\delta \varphi_{1}}+\frac{\delta}{\delta \varphi_{2}}+\frac{\delta}{\delta \varphi_{3}}\right)^{3} \gamma_{1} \gamma_{2} \gamma_{3} \\
& =e^{L_{123}+\left(L_{12}+L_{13}+L_{23}\right)+\left(L_{1}+L_{2}+L_{3}\right)} \gamma_{1} \gamma_{2} \gamma_{3}
\end{aligned}
$$

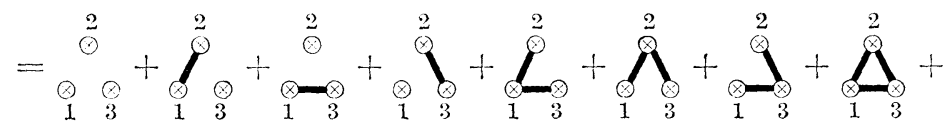

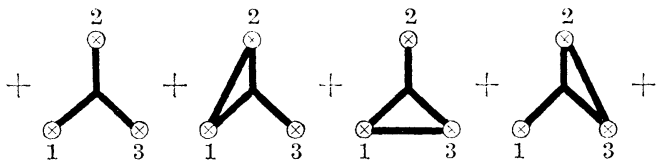

$$
\begin{aligned}
& +\prod_{0}^{2}+\prod_{3}^{2}+\underbrace{2}_{3}
\end{aligned}
$$


Bei dem in $\exp L \exp \gamma$ benötigten Übergang zu identischen Funktionalen $\gamma_{1}=\gamma_{2}=\gamma_{3}$ vereinfacht sich (5) wieder, da dann alle Cluster gleicher Struktur auch die gleiche mathematische Bedeutung aufweisen. Alle topologischen Überlegungen von Abschnitt 12 können sinngemäß übernommen werden, woraus sofort das Verbundclustertheorem auch für die Dreier-Kontraktionen folgt:

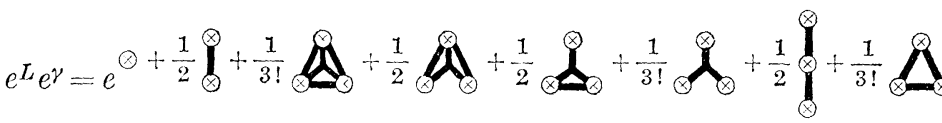

Die dabei auftretende Verbundcluster-Summe spielt dann als Funktional von $\varphi$ bei dem im nächsten Schritt herzustellenden Vierer-Kontraktionen die Rolle des elementaren Bausteins.

Die systematische Fortführung des geschilderten Vorgehens führt zu immer komplizierter werdenden Clustern und liefert schließlich das allgemeine Exponential-Verbundcluster-Theorem

$$
\begin{aligned}
e^{g\left(\frac{\delta}{\delta \varphi}\right)} e^{f(\varphi)} & =e^{g f(\varphi)} \\
g f(\varphi) & =\left(e^{g\left(\frac{\delta}{\delta \varphi}\right)} e^{f(\varphi)}\right) \text { Verbund } \\
& =\sum_{r_{1}, r_{2}, \ldots}\left(e^{g\left(\frac{\delta}{\delta \varphi}\right)} \frac{\left(\frac{\varphi}{1 !} f_{1}\right)^{r_{1}}}{r_{1} !} \frac{\left(\frac{\varphi^{2}}{2 !} f_{2}\right)^{r_{2}}}{r_{2} !} \cdots\right)_{\text {Verbund }}
\end{aligned}
$$

mit $g f(\varphi)$ als der Summe aller aus den Koeffizientenfunktionen $g_{s}, f_{r}$ konstruierten Verbundterme. Dabei werden also in dem Produkt $(\varphi f)^{r_{1}}\left(\varphi^{2} f_{2}\right)^{r_{2}} \ldots$ durch den Differentialoperator $\exp g(\delta / \delta \varphi)$ sukzessive einzelne, an allen möglichen Stellen stehende Feldgrößen, alle möglichen Paare, Tripel, Quadrupel von Feldgrößen usw. durch $g_{1}, g_{2}, g_{3}, g_{4}$ usw. ersetzt, aber so, daß nur verbundene Terme entstehen. Die Aussagen des Exponential-Verbundcluster-Theorems bestehen also darin, daß aus der Verbundeigenschaft der Funktionale $g(.$.$) und f(.$.$) die Verbund-$ eigenschaft des Funktionals $g f(.$.$) folgt und daß sich die Koeffizienten-$ funktionen von $g f(\ldots)$ in ganz bestimmter Weise aus denen von $g(\ldots)$ und $f(.$.$) aufbauen { }^{3}$. Gibt man die in (11.1) angegebenen Taylor/VolterraReihenfolge von $g$ und $f$ durch Graphen wieder

$$
(\hookleftarrow \equiv \delta / \delta \varphi, \leftrightarrow \equiv \varphi),
$$

3 Die letztere Aussage gilt übrigens auch dann, wenn $g(\ldots)$ und $f(\ldots)$ und damit auch $g f(. .$.$) die Verbundseigenschaft nicht besitzen.$ 
so lautet (7)

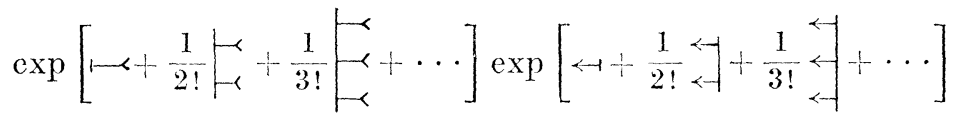

$$
\begin{aligned}
& =\exp \left[\left(H+\frac{1}{2 !} H_{H}+\frac{1}{2 ! H}+\frac{1}{2 !} H_{H}+H+\cdots\right)+\right.
\end{aligned}
$$

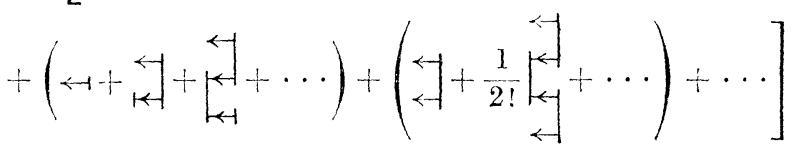

Eine mathematische Verallgemeinerung, die aber für physikalische Anwendungen bislang keine Rolle spielt, entsteht dadurch, daß man den Differentialoperator $\delta / \delta \varphi$ nicht nur auf $f(\varphi)$, sondern weiter nach rechts wirken läßt. Schließlich könnte $g$ noch Feldgrößen $\varphi$ und $f$ noch Differentialoperatoren $\delta / \delta \varphi$ enthalten:

$$
\left[N e^{g\left(\varphi, \frac{\delta}{\delta \varphi}\right)}\right]\left[N e^{f\left(\varphi, \frac{\delta}{\delta \varphi}\right)}\right]=N e^{g f\left(\varphi, \frac{\delta}{\delta \varphi}\right)}, \quad N \equiv N_{\varphi, \delta / \delta \varphi}
$$

Auch dann besitzt das so definierte Funktional $g f(. .$.$) ebenfalls die$ Verbundeigenschaft. Bei all diesen Manipulationen ist natürlich angenommen, daß die Funktionale $g(\ldots)$ und $f(\ldots)$ hinreichend, ,vernünftig" sind, so daß die hier vorgenommenen Reihenumordnungen nicht durch mangelnde Konvergenzeigenschaften behindert werden.

Einfachstes, nicht-triviales Beispiel für (7) ist die identische Umformung (mit $g_{2}=a, f_{2}=b$ )

$$
\begin{aligned}
F(\varphi)=e^{\frac{1}{2} a \frac{\delta^{2}}{\delta \varphi^{2}}} e^{\frac{1}{2} \varphi^{2} b} & =e^{\frac{1}{2} \operatorname{Sp}\left[a b+\frac{1}{2}(a b)^{2}+\frac{1}{3}(a b)^{3}+\cdots\right]+\frac{1}{2} \varphi^{2}[b+b a b+\cdots]} \\
& =e^{-\frac{1}{2} \operatorname{Sp} \ln (1-a b)+\frac{1}{2} \varphi^{2}(1-b a)^{-1} b}
\end{aligned}
$$

die man übrigens in diesem einfachen Fall auch unabhängig vom Verbundcluster-Theorem mit seinen Reihenentwicklungen sofort in geschlossener Form durch Lösung der Funktionaldifferentialgleichungen

$$
\left[(1-b a) \frac{\delta}{\delta \varphi}\right]_{x} F=(b \varphi)_{x} F \quad a \frac{\delta}{\delta a} F=\frac{1}{2} a \frac{\delta^{2}}{\delta \varphi^{2}} F
$$

erhält [12-14].

${ }^{4}$ Hier ordnet $N_{\varphi, \delta / \delta \varphi}$ alle $\varphi$ nach links und alle $\delta / \delta \varphi$ nach rechts.

${ }^{5}$ Hier wird ergänzend zu (11.2) die Schreibung eingeführt:

$$
\begin{aligned}
(b \varphi)_{x} & =\int d x^{\prime} b\left(x, x^{\prime}\right) \varphi\left(x^{\prime}\right), \quad\left(\frac{\delta}{\delta \varphi}\right)_{x}=\frac{\delta}{\delta \varphi(x)}, \\
\left(b a \frac{\delta}{\delta \varphi}\right)_{x} & =\int d x^{\prime} d x^{\prime \prime} b\left(x, x^{\prime}\right) a\left(x^{\prime}, x^{\prime \prime}\right) \frac{\delta}{\delta \varphi\left(x^{\prime \prime}\right)} .
\end{aligned}
$$


Anwendung findet das Verbundcluster-Theorem (7) in der Quantenelektrodynamik, in der klassischen $[15,16]$ und quantenmechanischen Gleichgewichts-Statistik und bei der Behandlung von Elektron-PhononSystemen sowie von Fermisystemen im Grundzustand, aber auch in der klassischen und quantenmechanischen Nichtgleichgewichts-Statistik bei der Entwicklung einer allgemeinen Korrelationsdynamik [17 - 19].

\section{Allgemeine Kontraktionsaufgabe mit mehreren Operatoren}

\subsection{Formulierung der Aufgabe und schrittweise Lösung}

Hier wird die Aufgabe

$$
e^{g\left(\frac{\delta}{\delta \psi}, \frac{\delta}{\delta \psi}\right)} e^{f(\bar{\psi}, \psi)}=e^{\sum_{s} \frac{1}{s !} \frac{\delta s}{\delta \psi^{s}} g_{s} \frac{1}{\delta} \psi^{s}} e^{\sum \frac{1}{r !} \bar{\psi}^{r} f_{r} \psi^{r}}
$$

behandelt. Dabei wird wieder konsequent die Abkürzung

$$
\begin{aligned}
\bar{\psi}^{r} f_{r} \psi^{r} \equiv \int d x_{1} d x_{1}^{\prime} \ldots & \int d x_{r} d x_{r}^{\prime} \bar{\psi}\left(x_{1}\right) \ldots \bar{\psi}\left(x_{r}\right) \times \\
& \times f_{r}\left(x_{1}, \ldots, x_{r} ; x_{r}^{\prime}, \ldots, x_{1}^{\prime}\right) \psi\left(x_{r}^{\prime}\right) \ldots \psi\left(x_{1}^{\prime}\right)
\end{aligned}
$$

verwendet. Je nachdem, ob mit $\bar{\psi}, \psi$ Fermionen oder Bosonen beschrieben werden, treten beim Vertauschen von Feldgrößen Vorzeichenwechsel auf oder nicht. Diese Regel überträgt sich übrigens auch auf die zugehörigen Funktionaldifferentialoperatoren. Bis auf etwaige Vorzeichenwechsel sind die Feldgrößen vertauschbar, da sie, wie hier nicht weiter angegeben, hinter entsprechenden Ordnungsoperatoren stehen. Aus Vorzeichengründen sind auch die Operatoren in (2) ,,paarweise“" angeordnet.

Auch hier kann die Aufgabe wieder schrittweise behandelt werden:

$$
e^{g\left(\frac{\delta}{\delta \psi}, \frac{\delta}{\delta \bar{\psi}}\right)} e^{f(\bar{\psi}, \psi)}=\cdots e^{\frac{1}{2 !} \frac{\delta^{2}}{\delta \psi^{2}} g_{2} \frac{\delta^{2}}{\delta \bar{\psi}^{2}}} e^{\frac{\delta}{\delta \psi} g_{1} \frac{\delta}{\delta \bar{\psi}}} e^{f(\bar{\psi}, \psi)}=\cdots e^{L} e^{K} e^{f(\bar{\psi}, \psi)}
$$

Zunächst verknüpft der bilineare Differentialoperator

$$
K \equiv \frac{\delta}{\delta \psi} g_{1} \frac{\delta}{\delta \bar{\psi}}= \pm \operatorname{Spg} g_{1} \frac{\delta}{\delta \bar{\psi}} \frac{\delta}{\delta \psi}
$$

in $\exp f(\bar{\psi}, \psi)$ alle möglichen Paare $\psi_{1} \equiv \psi\left(x_{1}\right), \bar{\psi}_{2} \equiv \bar{\psi}\left(x_{2}\right)$

$$
\widehat{\psi_{1} \bar{\psi}_{2}}=K \psi_{1} \bar{\psi}_{2}= \pm g_{1}(1 ; 2) \text {. }
$$

Dabei liefern die Kontraktionen innerhalb eines Funktionals $f(\bar{\psi}, \psi)$

$$
e^{K} f(\bar{\psi}, \psi) \equiv \bar{f}(\bar{\psi}, \psi) \equiv 0
$$

das Funktional $\vec{f}(\bar{\psi}, \psi)$ als Bauelement für die Kontraktionen in einem Produkt $f_{1} f_{2} \ldots$, für das wieder wegen der Produktregel für Differential-

${ }^{6}$ Das obere Vorzeichen bezieht sich stets auf Bosonen, das untere auf Fermionen. 
operatoren das verallgemeinerte Wicksche Theorem

$$
\begin{aligned}
& e^{K} f_{1} \ldots f_{n}=e^{\sum_{i<j}\left(K_{i j}+K_{j i}\right)} \vec{f}_{1} \ldots \bar{f}_{n} \\
& =\prod_{i<j}\left(1+\overparen{T}_{i j}\right) \vec{f}_{1} \ldots \vec{f}_{n}=\sum \vec{f}_{1} \cdots \vec{f}_{n} \quad K_{i j}=\widetilde{\psi} \bar{\psi} \frac{\delta^{2}}{\delta \bar{\psi}_{i} \delta \psi_{j}}
\end{aligned}
$$

entsteht. Daher können auch alle topologischen Überlegungen von Abschnitt 12 sofort übernommen werden, so daß wiederum

$$
e^{K} e^{f}=e^{\gamma} \quad \gamma \quad \gamma=\gamma(\bar{\psi}, \psi)=0+\frac{1}{2} \boldsymbol{g}+\frac{1}{3 !} \boldsymbol{\Delta}+\frac{1}{2} \boldsymbol{8}+\cdots
$$

gilt mit $\gamma(\bar{\psi}, \psi)$ als Summe aller aus Paarkontraktionen (5) aufgebauten Cluster. Beim nächsten Schritt dient $\gamma(\bar{\psi}, \psi)$ als Bauelement. Das Vorgehen ist ganz analog zu Abschnitt 12 und liefert auch hier das Exponential-Verbundcluster-Theorem

$$
\begin{gathered}
e^{g\left(\frac{\delta}{\delta \psi}, \frac{\delta}{\delta \bar{\psi}}\right)} e^{f(\bar{\psi}, \psi)}=e^{g f(\bar{\psi}, \psi)} \\
g f(\bar{\psi}, \psi)=\left(e^{g\left(\frac{\delta}{\delta \bar{\psi}}, \frac{\delta}{\delta \bar{\psi}}\right)} e^{j(\bar{\psi}, \psi)}\right)_{\text {Verbund }} \\
=\sum_{r_{1}, r_{2}, \ldots}\left(e^{g\left(\frac{\delta}{\delta \psi}, \frac{\delta}{\delta \bar{\psi}}\right)} \frac{\left(\frac{1}{1 !} \bar{\psi} f_{1} \psi\right)^{r_{1}}}{r_{1} !} \frac{\left(\frac{1}{2 !} \bar{\psi}^{2} f_{2} \psi^{2}\right)^{r_{2}}}{r_{2} !} \cdots\right)_{\text {Verbund }}
\end{gathered}
$$

Dieses Theorem gilt ebenfalls für die zu (12.8) analoge Verallgemeinerung sowie für den Fall, daß die Funktionale $g(\ldots)$ und $f(\ldots)$ bei ihrer Taylor/Volterra-Entwicklung auch, ,nichtdiagonale“ Terme $\bar{\psi}^{r} f_{r r^{\prime}} \psi^{r^{\prime}}$ mit $r \neq r^{\prime}$ enthalten.

Einfachstes Beispiel für (9) ist die Beziehung $\left(g_{1}=a, f_{1}=b\right)$

$$
F(\bar{\psi}, \psi)=e^{\frac{\delta}{\delta \psi} a \frac{\delta}{\delta \bar{\psi}}} e^{\bar{\psi} b \psi}=e^{\mp \operatorname{Sp} \ln (1 \mp a b)+\bar{\psi}(1 \mp b a)^{-1} b \psi},
$$

die ebenfalls durch Lösung der Funktional-Differentialgleichungen

$$
\left[(1 \mp b a) \frac{\delta}{\delta \bar{\psi}}\right]_{x} F=(b \psi)_{x} F \quad a \frac{\delta}{\delta a} F=\frac{\delta}{\delta \psi} a \frac{\delta}{\delta \bar{\psi}} F
$$

gewonnen werden kann [12-14].

Von dem Verbundcluster-Theorem (9) wird in der Gleichgewichtsund Nichtgleichgewichts-Statistik [15-19] sowie in der relativistischen und nichtrelativistischen Quantenfeldtheorie ausgiebig Gebrauch gemacht. 
Schließlich sei noch die Verallgemeinerung von (9) bzw. (12.8) auf mehrere Differentialoperatoren angegeben:

$$
\begin{gathered}
{\left[N e^{g\left(\alpha, \beta, \gamma, \ldots ; \ldots \frac{\delta}{\delta \gamma}, \frac{\delta}{\delta \beta}, \frac{\delta}{\delta \alpha}\right)}\right]\left[N e^{f\left(\alpha, \beta, \gamma, \ldots ; \ldots \frac{\delta}{\delta \gamma}, \frac{\delta}{\delta \bar{\beta}}, \frac{\delta}{\delta \alpha}\right)}\right]} \\
=N e^{g f\left(\alpha, \beta, \gamma, \ldots ; \ldots \frac{\delta}{\delta \gamma}, \frac{\delta}{\delta \beta}, \frac{\delta}{\delta \alpha}\right)} \\
N \equiv N_{\alpha, \beta, \gamma, \ldots ; \ldots \delta / \delta \gamma, \delta / \delta \beta, \delta / \delta \alpha} .
\end{gathered}
$$

Das Verbundgraphentheorem besagt nun, daß aus der Verbundeigenschaft von $g(\ldots)$ und $f(\ldots)$ diejenige von $g f(\ldots)$ folgt.

\subsection{Wicksches Theorem}

Häufigste Anwendung finden die in den vorangehenden Abschnitten angegebenen Verbundgraphen-Theoreme im Zusammenhang mit dem Wickschen Theorem. Letzteres macht eine Aussage über Umordnungen von Operatorenprodukten. Meist gilt es, die Zeitordnung $T \equiv N_{t>}$, durch die alle Feldoperatoren $\bar{\psi}, \psi, \varphi$ mit nach links zunehmender Zeit geordnet werden, in die sog. Normalordnung $N \equiv N_{+-}$zu überführen, bei der alle Feldoperatoren $\bar{\psi}, \psi, \varphi$ in einen Positiv- und einen Negativ-Frequenzteil zerlegt und diese Anteile entsprechend geordnet werden. Diese Normalordnung ist stets an einen bestimmten Zustand $><$ angepaßt, mit dem alle Erwartungswerte gebildet werden und für den die daher nützliche Vakuumeigenschaft $\psi^{-}>=\bar{\psi}^{-}>=\varphi^{-}>=0$ und $\left\langle\psi^{+}=<\bar{\psi}^{+}=<\varphi^{+}=0\right.$ gilt. Bei dieser Umordnung entsteht durch jedes Operatorenpaar $\psi_{1}, \bar{\psi}_{2}$ bzw. $\varphi_{1}, \varphi_{2}$ ein $c$-Zahl-Beitrag

$$
\begin{aligned}
& \overparen{\psi_{1} \bar{\psi}_{2}}=(T-N) \psi_{1} \bar{\psi}_{2}=\theta_{12}\left[\psi_{1}^{-}, \bar{\psi}_{2}^{+}\right]_{+}-\theta_{21}\left[\bar{\psi}_{2}^{-}, \psi_{1}^{+}\right]_{+}=\left\langle T \psi_{1} \bar{\psi}_{2}\right\rangle \\
& \bar{\varphi}_{1} \varphi_{2}=(T-N) \varphi_{1} \varphi_{2}=\theta_{12}\left[\varphi_{1}^{-}, \varphi_{2}^{+}\right]_{-}+\theta_{21}\left[\varphi_{2}^{-}, \varphi_{1}^{+}\right]_{-}=\left\langle T \varphi_{1} \varphi_{2}\right\rangle .
\end{aligned}
$$

Dieser als Wicksches Theorem bezeichnete Sachverhalt wird nach HorI, ANDERson und Matsubara kompakt mit Funktionalableitungen wiedergegeben [2-4]; für die $T$ - $N$-Umordnung lautet es

$T=N e^{\overline{\psi \bar{\psi}} \frac{\delta^{2}}{\delta \bar{\psi} \delta \psi}+\frac{1}{2} \overrightarrow{\varphi \varphi} \frac{\delta^{2}}{\delta \varphi \delta \varphi}}$
$\widehat{\psi}_{1} \bar{\psi}_{2}=(T-N) \psi_{1} \bar{\psi}_{2}, \widehat{\varphi}_{1} \varphi_{2}=(T-N) \varphi_{1} \varphi_{2},\langle N F(\bar{\psi}, \psi, \varphi)\rangle=F(0,0,0)$.

In der Gleichgewichts-Statistik gilt es oft, die Temperaturordnung $N_{\beta>}$, durch die alle Feldoperatoren mit nach links zunehmender reziproker Temperatur $\beta=1 / k T$ geordnet werden, in eine Ordnung $N_{+-}$zu überführen, die nach Matsubara [4] im Sinne von $\operatorname{Sp}^{0} N_{+_{-}} F(\bar{\psi}, \psi, \varphi)=0$ an die Spurbildung mit dem ungestörten statistischen Operator $\varrho^{0}$ angepaßt ist; weiter spielen die Umordnungen $N_{\beta>} \rightarrow N_{\bar{\psi}, \psi}$ und $N_{\psi, \bar{\psi}} \rightarrow N_{\bar{\psi}, \psi}$ 
eine Rolle. Für sie gelten zu (2) ganz analoge Theoreme, aus denen bei Anwendung auf Exponentialfunktionale der Zusammenhang mit den vorangehenden Abschnitten unmittelbar hervorgeht.

Gelegentlich interessiert nicht wie in (2) die $T-N$-Umordnung, sondern die $1-N$-Umordnung, d. h. die Herstellung der Normalordnung $N=N_{+-}$in einfachen (statt zeitgeordneten) Produkten. Dann tritt - bei Anwendung auf Exponential-Funktionale - an die Stelle von (2) die von (11.1) und (21.1) etwas verschiedene Aufgabe

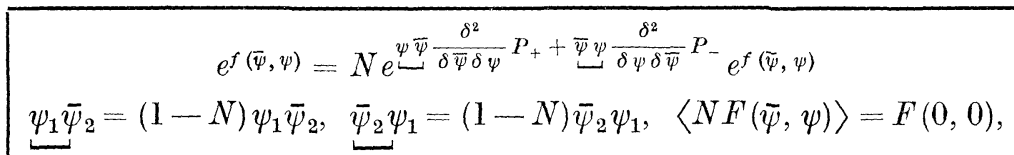

wobei Projektionsoperatoren $P_{ \pm}$

$$
P_{ \pm} \bar{\psi} \psi=\left\{\begin{array}{l}
0 \\
\bar{\psi} \psi
\end{array} \quad P_{ \pm} \psi \bar{\psi}=\left\{\begin{array}{l}
\psi \bar{\psi} \\
0
\end{array}\right.\right.
$$

dafür sorgen, daß je nach der Anordnung der Operatoren die richtigen Kontraktionen entstehen. Auch für (3) gilt ein Exponential-Verbundcluster-Theorem, was im folgenden für den in der GleichgewichtsStatistik häufig benötigten Sonderfall von (3), nämlich $N=N_{\bar{\psi}, \psi}$, also $\bar{\psi} \psi=0$ abschließend noch bewiesen werden soll:

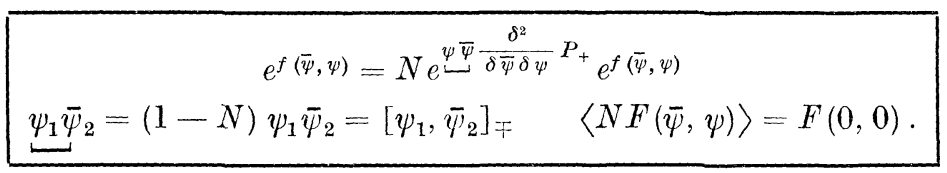

Da $f(\bar{\psi}, \psi)$ schon normalgeordnet ist, treten Kontraktionen nur zwischen verschiedenen Faktoren auf. Dabei entstehen jedoch Cluster

$$
\begin{aligned}
& N e^{K} f_{1} f_{2}=N\left(f_{1} f_{2}+f_{1} f_{2}\right)=N\left(\begin{array}{ll}
0 & 0 \\
1 & 2
\end{array}+\underset{1}{\infty}\right) \quad K=\psi \bar{\psi} \frac{\delta^{2}}{\delta \bar{\psi} \delta \psi} P
\end{aligned}
$$

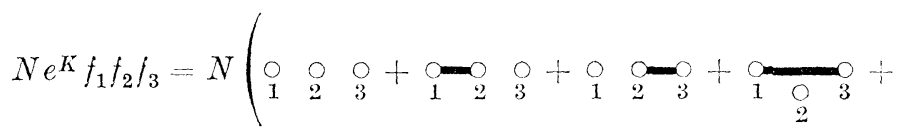

$$
\begin{aligned}
& \left.+\underset{1=3}{\alpha-m_{3}}+\sum_{2}^{3}+\underset{2}{2}+{\underset{1}{2}}_{3}^{2}\right) \text {, }
\end{aligned}
$$

die von (12.9) deswegen etwas verschieden sind, weil die hier zu ordnenden Ausdrücke nicht zeitgeordnete Produkte $T f_{1} f_{2} \ldots$, in denen die Faktoren $f_{1}, f_{2} \ldots$ vertauschbar sind, sondern einfache Produkte $f_{1} f_{2} \ldots$ darstellen, in denen die Faktoren $f_{1}, f_{2}, \ldots$ natürlich nicht vertauschbar sind, so daß bei der Clusterbildung an der einmal gewählten FaktorenReihenfolge $f_{1} f_{2} \ldots$ starr festzuhalten ist. Beim Übergang zu identischen 
Funktionalen $f_{1}=f_{2}=\cdots$ entstehen daher auch im Vergleich zu (12.10) mehr voneinander verschiedene Cluster, die sich von (12.10) durch die Anordnung der Punkte etwas unterscheiden

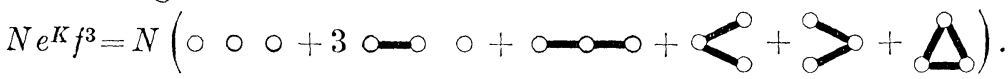

Die an (12.10) anschließenden kombinatorischen Überlegungen können aber wieder sofort übernommen werden, so daß auch hier ein Exponential-Verbundcluster-Theorem

$$
\begin{aligned}
& N e^{\psi} \stackrel{\psi \bar{\psi}}{\frac{\delta^{2}}{\partial \bar{\psi} \delta \psi} P_{+}} e^{j\left(\bar{\psi}, \psi^{\prime}\right)}=N e^{\gamma(\bar{\psi}, \psi)} \\
& \begin{aligned}
\gamma(\bar{\psi}, \psi)=0+\frac{1}{2} & \infty+1 \\
& +\frac{1}{3 !}(\boldsymbol{\Delta}+\infty+\infty+\infty+\infty)+\cdots
\end{aligned}
\end{aligned}
$$

gilt mit $\gamma(\bar{\psi}, \psi)$ als der Summe aller zu $f(\bar{\psi}, \psi)$ gehörigen Verbundcluster der $1-N_{\bar{\psi}, \psi^{-}}$Umordnung.

Speziell für Einteilchen-Exponential-Funktionale $f=\psi^{\dagger} u \psi$ läßt sich die Verbundcluster-Summe in (8) explizit und geschlossen angeben ${ }^{7}$. Mit $\left[\psi_{1}, \psi_{2}^{\dagger}\right]_{\mp}=\delta\left(x_{1}-x_{2}\right)$ ergibt sich nämlich $\gamma\left(\psi^{\dagger}, \psi\right)$ sofort zu $\gamma\left(\psi^{\dagger}, \psi\right)=\psi^{\dagger} u \psi+\frac{1}{2 !} \psi^{\dagger} u \psi \psi^{\dagger} u \psi+\frac{1}{3 !} \psi^{\dagger} u \psi \psi^{\dagger} u \psi \psi^{\dagger} u \psi+\cdots$

$=\psi^{\dagger}\left(e^{u}-1\right) \psi=\int d x d x^{\prime} \psi^{\dagger}(x)\left(e^{u}-1\right)_{x x^{\prime}} \psi\left(x^{\prime}\right), \quad \underline{\psi}_{1}^{\psi_{2}^{\dagger}}=\delta\left(x_{1}-x_{2}\right)$,

da $n$ hintereinanderstehende Bauelemente $\psi^{\dagger} u \psi$ nur genau eine VerbundKontraktion ermöglichen, so daß die zugehörigen Symmetriefaktoren $z=1$ und $g=n$ ! lauten. Es gelten daher die in der GleichgewichtsStatistik wiederholt benötigten Beziehungen

$$
e^{\psi^{\dagger} u \psi}=N_{\psi}{ }^{\dagger}, \psi e^{\psi^{\dagger}\left(e^{u}-1\right) \psi} \quad N_{\psi^{\dagger}, \psi} e^{\psi^{\dagger} v \psi}=e^{\psi^{\dagger} \ln (1+v) \psi} .
$$

Die zweite Gleichung folgt mit $v=(\exp u)-1$, also $u=\ln (1+v)$ sofort aus der ersten. Ein Potenzvergleich in (10) liefert $\left(\delta_{s^{\prime}-s}\right.$ bezeichnet das Kroneckersymbol)

$$
\begin{aligned}
\frac{\left(\psi^{\dagger} u \psi\right)^{s}}{s !}=N_{\psi^{\dagger}, \psi} \sum_{s_{1}, s_{2}, \ldots} & \delta_{s_{1}+2 s_{2}+\cdots-s} \times \\
& \times \frac{\left(\frac{1}{1 !} \psi^{\dagger} u \psi\right)^{s_{1}}}{s_{1} !} \frac{\left(\frac{1}{2 !} \psi^{\dagger} u^{2} \psi\right)^{s_{2}}}{s_{2} !} \frac{\left(\frac{1}{3 !} \psi^{\dagger} u^{3} \psi\right)^{s_{3}}}{s_{3} !} \ldots \\
N_{\psi^{\dagger}, \psi} \frac{\left(\psi^{\dagger} v \psi\right)^{s}}{s !} \sum_{s_{1}, s_{2}, \ldots} \delta_{s_{1}+2 s_{2}+\cdots-s} \times & \\
\times & \times \frac{\left(\frac{1}{1} \psi^{\dagger} v \psi\right)^{s_{1}}}{s_{1} !} \frac{\left(-\frac{1}{2} \psi^{\dagger} v^{2} \psi\right)^{s_{2}}}{s_{2} !} \frac{\left(\frac{1}{3} \psi^{\dagger} v^{3} \psi\right)^{s_{3}}}{s_{3} !} \ldots
\end{aligned}
$$

7 Hierbei werden nur nichtrelativistische, temperatur- und zeitunabhängige Feldoperatoren betrachtet, so daß $\psi^{\dagger}$ an die Stelle von $\bar{\psi}$ tritt. 
Für konstante Einteilchenfunktionen $u\left(x, x^{\prime}\right)=$ konst, $v\left(x, x^{\prime}\right)=$ konst geht (11) mit $\psi^{\dagger} \psi \equiv \int d x \psi^{\dagger}(x) \psi(x)$ in die einfacheren, auch durch vollständige Induktion beweisbaren Beziehungen

$$
\frac{\left(\psi^{\dagger} \psi\right)^{s}}{s !}=N_{\psi^{\dagger}, \psi}\left\{\begin{array}{c}
\psi^{\dagger} \psi \\
s
\end{array}\right\}, N_{\psi^{\dagger}, \psi} \frac{\left(\psi^{\dagger} \psi\right)^{s}}{s !}=\left(\begin{array}{c}
\psi^{\dagger} \psi \\
s
\end{array}\right)
$$

über. Die Ausdrücke in den runden Klammern sind dabei die bekannten, bei Entwicklung von $\exp \psi^{\dagger} \psi \ln (1+v)=(1+v)^{\psi^{\dagger} \psi}$ auftretenden Binomial-Koeffizienten, während die Ausdrücke in den geschweiften Klammern die Koeffizienten bedeuten, die bei der Entwicklung von $\exp \psi^{\dagger} \psi\left(e^{u}-1\right)$ nach Potenzen von $u$ auftreten (siehe Anhang).

\section{Schlußbemerkung}

Die Aufgabe, Produkte von Exponentialfunktionalen umzuordnen, kann auf die für Mehrteilchenkräfte verallgemeinerte Mayersche Clustertheorie der klassischen Gleichgewichts-Statistik zurückgeführt werden. Das Ergebnis ist das allgemeine Exponential-Verbundgraphen-Theorem (21.12). Über dessen Anwendungen in der Gleichgewichts- und Nichtgleichgewichts-Statistik, in der relativistischen und nichtrelativistischen Quantenfeldtheorie, in der Schwankungstheorie und in der Kohärenztheorie wird in weiteren Arbeiten berichtet.

Herrn Prof. Dr. W. Macke danke ich für anregende Diskussionen sowie für die Unterstützung dieser Arbeit durch Gewährung von Arbeitsmöglichkeiten am Institut für Theoretische Physik der Technischen Universität Dresden.

\section{Anhang}

\section{Binomial- und Biexponentialkoeffizienten}

Bei der Entwicklung des Ausdrucks

$$
(1+\alpha)^{x}=e^{x \ln (1+\alpha)}=e^{x\left(\frac{\alpha}{1}-\frac{\alpha^{2}}{2}+\cdots \cdots\right)}=\sum_{s}\left(\begin{array}{l}
x \\
s
\end{array}\right) \alpha^{s}
$$

nach Potenzen von $\alpha$ treten bekanntlich die sog. Binomialkoeffizienten

$$
\left.\left(\begin{array}{l}
x \\
s
\end{array}\right)=\sum_{s_{1} s_{2} \ldots} \delta_{s_{1}+2 s_{2}+\cdots-s} \frac{\left(\frac{1}{1}\right)^{s_{1}}\left(-\frac{1}{2}\right)^{s_{2}}}{s_{1} !} \cdots x^{s_{1}+s_{2}+\cdots}=\frac{1}{s !} \sum_{r(\leqq s)}\left(\begin{array}{l}
r \\
s
\end{array}\right)\right) x^{r}
$$

auf, die wiederum ihrerseits bei Entwicklung nach $x$ die Koeffizienten

$$
\left(\left(\begin{array}{l}
r \\
s
\end{array}\right)\right)=s ! \sum_{s_{1} s_{2} \ldots} \delta_{s_{1}+2 s_{2}+\cdots-s} \delta_{s_{1}+s_{2}+\cdots-r} \frac{\left(\frac{1}{1}\right)^{s_{1}}}{s_{1} !} \frac{\left(-\frac{1}{2}\right)^{s_{2}}}{s_{2} !} \cdots
$$

enthalten. So gilt $\mathrm{z} . \mathrm{B}$.

$$
\left(\begin{array}{l}
x \\
3
\end{array}\right)=\frac{x^{3}-3 x^{2}+2 x}{3 !} \sim\left(\left(\begin{array}{l}
3 \\
3
\end{array}\right)\right)=1\left(\left(\begin{array}{l}
2 \\
3
\end{array}\right)\right)=-3\left(\left(\begin{array}{l}
1 \\
3
\end{array}\right)\right)=2 .
$$


Ähnlich treten bei der Entwicklung des Ausdrucks

$$
\left(e^{\left(e^{\alpha}-1\right)}\right)^{x}=e^{x\left(e^{\alpha}-1\right)}=e^{x\left(\frac{\alpha}{1 !}+\frac{\alpha^{2}}{2 !}+\cdots\right)}=\sum_{s}\left\{\begin{array}{l}
x \\
s
\end{array}\right\} \alpha^{s}
$$

nach Potenzen von $\alpha$ die hier als Biexponentialkoeffizienten bezeichneten

$$
\begin{aligned}
& \text { Zahlen } \\
& \left.\left\{\begin{array}{l}
x \\
s
\end{array}\right\}=\sum_{s_{1} s_{2} \ldots} \delta_{s_{1}+2 s_{2}+\cdots-s} \frac{\left(\frac{1}{1 !}\right)^{s_{1}}}{s_{1} !} \frac{\left(\frac{1}{2 !}\right)^{s_{2}}}{s_{2} !} \cdots x^{s_{1}+s_{2}+\cdots}=\frac{1}{s !} \sum_{r(\leqq s)}\left\{\begin{array}{l}
r \\
l s
\end{array}\right)\right\} x^{r}
\end{aligned}
$$

auf, die ihrerseits bei Entwicklung nach $x$ die Koeffizienten

$$
\left\{\left\{\begin{array}{l}
r \\
s\}
\end{array}\right\}=s ! \sum_{s_{1} s_{2} \ldots} \delta_{s_{1}+2 s_{2}+\cdots-s} \delta_{s_{1}+s_{2}+\cdots-r} \frac{\left(\frac{1}{1 !}\right)^{s_{1}}}{s_{1} !} \frac{\left(\frac{1}{2 !}\right)^{s_{2}}}{s_{2} !} \cdots\right.
$$

enthalten. So gilt z. B.

$$
\left.\left\{\begin{array}{l}
x \\
3
\end{array}\right\}=\frac{x^{3}+3 x+x}{3 !} \neg\left\{\left\{\begin{array}{l}
3 \\
3
\end{array}\right\}\right\}=1 \quad\left\{\begin{array}{l}
2 \\
(3
\end{array}\right\}\right\}=3 \quad\left\{\left\{\begin{array}{l}
1 \\
3
\end{array}\right\}\right\}=1 .
$$

Weitere Beispiele für (7) sind

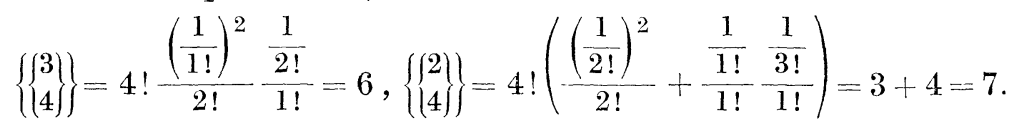

Mit der an ein Laplacesches Dreieck erinnernden Rekursionsformel

$\left(\frac{1}{x} \frac{\partial}{\partial \alpha}-1-\frac{\partial}{\partial x}\right) e^{x\left(e^{\alpha}-1\right)}=0 \frown\left\{\left\{\begin{array}{l}r+1 \\ s+1\end{array}\right\}\right\}=\left\{\left\{\begin{array}{l}r \\ s\end{array}\right\}\right\}+(r+1)\left\{\left\{\begin{array}{c}r+1 \\ s\end{array}\right\}\right\}$

können die höheren Koeffizienten sukzessive aus den niederen bestimmt werden.

\section{Literatur}

1. Wrck, G. C.: Phys. Rev. 80, 268 (1950).

2. Hori, S.: Progr. Theor. Phys. 7, 578 (1952).

3. Anderson, J. L.: Phys. Rev. 94, 703 (1955).

4. Matsubara, T.: Progr. Theor. Phys. 14, 351 (1955).

5. Heffener, H., and W. H. Louiselle: J. Math. Phys. 6, 474 (1965).

6. Bogoljubow, N. N.: J. Phys. (USSR) 10, 256 u. 265 (1946).

7. Schwinger, J.: Proc. Nat. Acad. Sci. 37, 452 u. 455 (1951).

8. Martin, P. C., and J. Schwinger: Bull. Am. Phys. Soc. 3, 202 (1959); Phys. Rev. 115, 1342 (1959).

9. Edwards, S. F., and R. E. Peierls: Proc. Roy. Soc. A 224, 24 (1954).

10. Symanzik, K.: Z. Naturforsch. 9a, 809 (1954).

11. Fradkin, E. S.: DAN 98, 47 (1954) u. 100, 897 (1955).

12. Thirring, E.: Vortrag während des Keszthely-Symposiums, Ungarn 1964.

13. Wess, J.: Vortrag während des Schladming-Symposiums, Österreich 1965.

14. Ziesche, P.: Acta Phys. Hung. 21, 219 (1966).

15. Schönberg, M.: Nuovo Cimento 9, 1139 (1952) u. 10, 419 u. 697 (1953).

16. Ziesche, P.: Commun. Math. Phys. 5, 191 (1967).

17. - Ann. Physik (in Vorbereitung).

18. Quads, P., K. Voss u. P. Ziesche: Acta Phys. Hung. (in Vorbereitung).

19. - - Ann. Phys. (in Vorbereitung).

20. Mayer, J. E.: J. Chem. Phys. 5, 67 (1937).

21. -, u. M. G. Mayer: Statistical mechanics. New York: Wiley 1940. 